\title{
L'incidence de la correction phonétique sur l'acquisition des voyelles en langue étrangère : étude de cas d'anglophones apprenant le français.
}

\author{
Charlotte Alazard-Guiu ${ }^{1}$, Fabian Santiago ${ }^{2}$, Paolo Mairano ${ }^{3}$ \\ (1) U.R.I Octogone-Lordat, Université de Toulouse, 31058 Toulouse, France \\ (2) UMR 7023, SFL, Université Paris 8, 75017, Paris, France \\ (3) LFSAG, Université de Turin, 10100, Turin, Italie \\ charlotte.alazardeuniv-tlse2.fr, fabian.santiago-vargas@univ-paris8.fr, \\ paolo.mairano@unito.it
}

\section{RÉSUMÉ}

Cet article a pour objectif d'étudier l'incidence de la correction phonétique sur l'acquisition des voyelles en L2. Des apprenants ont suivi des cours de correction phonétique selon deux méthodes différentes, la Méthode Verbo-Tonale et la Méthode Articulatoire. Dans une précédente étude (Alazard, 2013) nous avons comparé l'impact de chacune de ces méthodes sur le développement de la fluence. Dans cette étude, nous avons voulu tester l'incidence de la méthode utilisée sur les valeurs formantiques (F1-F2-F3) des voyelles produites par les apprenants avant et après les deux types d'entraînement. Nos premiers résultats montrent un effet du temps sur les valeurs formantiques de F3 mais pas d'effet de la méthode. Cette étude, bien que préliminaire, nous amène à penser que l'acquisition des voyelles en L2 met en jeu des processus spécifiques associés à une approche bimodale de la parole, que l'entrainement soit ou non explicitement focalisé sur les mouvements des lèvres.

\section{ABSTRACT \\ The incidence of phonetic correction on the acquisition of L2 vowels : a case study of L1 English learners of L2 French.}

This article aims at studying the incidence of phonetic correction on the acquisition of L2 segmental patterns. Foreign L2 French learners attended phonetic classes with two different methods: the Verbo-Tonal vs. the Articulatory method. In a previous study (Alazard, 2013), we compared the incidence of these two methods on the acquisition of L2 fluency. In this study, we measured vowel formants (F1-F2-F3) on learners' productions before and after training. Our first results show an effect of time on F3 but no effect of the method. This study, though preliminary, leads us to think that vowel acquisition implies specific processes associated with a bimodal approach of speech, even if the training does not explicitly focus on lip movements.

MOTS-CLÉS: Acquisition d'une langue étrangère, Phonétique corrective, Formants vocaliques

KEYWORDS: Second Language Acquisition, Phonetic correction, Vowel formants 


\section{Introduction}

Si l'oral a aujourd'hui une place reconnue dans l'enseignement du Français Langue Etrangère (désormais FLE), la place laissée à la phonétique corrective ne va pas de soi. On préfère généralement substituer à l'oral des quasi-synonymes, tels qu'interaction ou communication, qui ne rendent pas compte de sa nature même, et en particulier de sa dimension sonore. Ce constat peut en partie s'expliquer par l'existence de présupposés tenaces, notamment l'idée selon laquelle il n'est plus possible de maîtriser les aspects sonores d'une L2 après une période donnée ou encore l'idée selon laquelle la prononciation s'acquiert naturellement par des contacts répétés avec la langue cible.

Pourtant, des études montrent que des apprenants peuvent acquérir une prononciation perçue comme native grâce à un entrainement intensif en perception et/ou en production des sons de la langue étrangère (Bongaerts et al, 2000 ; Birdsong, 2003). En outre, dans une étude longitudinale (Alazard, 2013), nous avons comparé l'impact de deux méthodes de correction phonétique sur l'acquisition de la fluence en L2Nous avions retenu deux méthodes qui offraient deux approches opposées de l'apprentissage de la prononciation : une approche sans métalangage et plus 'implicite' avec la méthode verbo-tonale (MVT) et une approche descriptive et explicite avec la méthode articulatoire (ART).

La MVT se base en effet sur une approche procéduralisée de l'enseignement de la prononciation. Cette méthode utilise la structure prosodique de la langue cible comme support à l'acquisition de compétences phonologiques en L2. Plus spécifiquement, les patrons rythmiques de la langue cible sont utilisés pour mettre en lumière les spécificités de la langue cible (accentuation, intonation et phonèmes). L'enseignant va aider l'apprenant à se familiariser avec la structure prosodique de la langue cible à travers la répétition de logatomes (tels que « lalala » ou " dadada ») ou l'utilisation de gestes facilitateurs (par exemple un battement de la main pour souligner les proéminences). Dans une seconde phase, la structure prosodique et la gestualité accompagnatrice sont utilisées pour faciliter la perception et la reproduction des phonèmes. Par exemple, si l'apprenant assombrit le timbre du phonème cible, l'enseignant prononcera ledit phonème dans un contexte prosodique éclaircissant (c'est-à-dire à l'intérieur d'une syllabe accentuée), avec un geste de tension et demandera à l'apprenant de répéter le son dans ce nouveau contexte (Billières, 2005). La MVT se focalise sur un apprentissage non-explicite de la prononciation aidé par la gestuelle.

À l'opposé, la méthode articulatoire, postule qu'une bonne production implique la connaissance métalinguistique de l'articulation des sons, autrement dit, la mise en place de connaissances déclaratives. En conséquence, l'enseignant donne une description articulatoire des différents sons puis invite l'apprenant à répéter le geste articulatoire afin de produire le son cible. Par exemple, pour produire un /y/ l'enseignant dira à l'apprenant de placer sa langue à l'avant de sa bouche et d'arrondir les lèvres. Dans cette méthode, on met l'emphase sur la production et la répétition de sons isolés, puis de mots isolés contenant le son cible et finalement de phrases. Les paramètres prosodiques sont globalement négligés bien qu'une description métalinguistique des caractéristiques intonatives de la langue cible puisse être envisagée dans les pratiques de classes, le plus souvent en fin de formation.

Dans une précédente étude (Alazard 2013), nous avions montré que seul un entrainement à la correction phonétique par la MVT permettait à des apprenants débutants de développer une réelle compétence en fluence en L2, en seulement trois semaines. En effet, nous avions observé une diminution du nombre de pauses agrammaticales, une augmentation du taux d'articulation et une 
augmentation du débit de parole uniquement pour les apprenants qui avaient suivi ces cours de correction phonétique. De plus, nous avions observé, chez ces mêmes apprenants, une nette diminution de la durée des syllabes inaccentuées et une augmentation de la durée des syllabes accentuées entre le premier et le deuxième test, indiquant une augmentation de la densité accentuelle et du contraste accentuées/inaccentuées. Ces variations de durée accentuelles semblaient indiquer que les apprenants produisaient un schéma accentuel plus proche de celui du français après entrainement. De même, ils étaient également capables de produire des groupes rythmiques beaucoup plus longs. Cela nous avait amené à penser que les apprenants qui avaient suivi un entrainement via la MVT avaient commencé à mettre en place des stratégies d'encodage adaptées à la L2 via la maitrise des patrons prosodiques du français.

Dans cette étude, nous souhaitons mesurer les bénéfices de ces deux types d'entraînement, non plus sur les aspects prosodiques, mais sur la prononciation des voyelles. Nous pensons que la MVT pourrait avoir une plus grande incidence sur la différenciation des timbres vocaliques des voyelles en position accentuées, grâce à l'attention consacrée au niveau prosodique et à l'utilisation de procédés correctifs visant à modéliser l'aperture et le lieu d'articulation par des gestes de tension ou de relâchement.

\section{$2 \quad$ Méthodologie}

Pour comparer l'impact des deux méthodes et tester notre hypothèse, nous avons repris les données de l'étude précédente (Alazard 2013). Ces données avaient été recueillies via une étude longitudinale pilote, sur trois semaines, à raison de 2 séances de correction phonétique de 90 min par semaine. Tous les cours avaient été dispensés par le même enseignant, formé aux deux méthodes.

Nous avions enregistré huit apprenants anglophones de niveau débutant divisés en deux groupes selon la méthode suivie (ART vs MVT). Le niveau des apprenants avait été évalué pour l'étude sur la base d'un entretien oral semi-directif selon l'échelle proposée par le CECRL. Ce test de niveau, inspiré des épreuves d'évaluation de la production et de la compréhension orales du DELF-DALF, comprenait plusieurs activités : un entretien guidé, des répétitions de phrases et une activité de compréhension orale. Les participants étaient âgés de 20 à 60 ans (moyenne d'âge 32,5 ans). Tous les participants résidaient en France au moment de l'étude. Ils avaient tous préalablement suivi un enseignement en français, soit au cours de leur scolarité dans leur pays d'origine, soit à leur arrivée en France. En parallèle des cours proposés dans le cadre de cette recherche, la majorité des apprenants suivaient également des cours de français.

Les apprenants avaient été testés dans une tâche de lecture oralisée à deux reprises, avant la formation (T1) et après trois semaines d'entrainement exclusivement à l'oral (T2). Les textes concernaient des passages/courtes histoires adaptés au niveau des apprenants. Les sujets avaient passé les tests individuellement dans une salle d'enregistrement adaptée, à l'université de Toulouse 2. Ils avaient pour consigne de s'approprier le texte par une ou plusieurs lectures silencieuses avant d'être enregistrés. Après avoir pris connaissance du texte, les sujets lisaient une fois le texte à voix haute avant de répondre à des questions de compréhension posées par l'examinateur.

Les données recueillies avaient d'abord été transcrites phrase par phrase selon les conventions de la Transcription Orthographique Enrichie (TOE) (Bertrand et al, 2008). Les phrases transcrites avaient ensuite été alignées automatiquement grâce au logiciel SPPAS (Bigi \& Hirst, 2012) et 
segmentées à différents niveaux : mots et syllabes. Pour cette étude, les voyelles ont été réétiquetées et réalignées manuellement dans Praat (Boersma \& Weenink, 2005).

\section{$3 \quad$ Annotation et analyse formantique des voyelles}

\subsection{Voyelles analysées}

L'ensemble de notre corpus contenait 2704 segments vocaliques produits dans les tâches de lecture par les participants, dont 1361 voyelles produites avant la formation (T1), et 1340 après l'entraînement (T2). Nous avons utilisé une transcription canonique des mots, et non une transcription de la production réelle de l'apprenant (p. ex. pour le mot < déjeuner $>$, la transcription était [dezœne], même si l'apprenant prononçait [dəzune]). Cela nous a permis de vérifier dans quelle mesure les formants des voyelles ciblées changeaient (ou pas) en fonction de la méthode employée/après entrainement.

Nous avons évalué deux types de métriques : la première consistait à analyser le timbre des voyelles [i], [y] [e], [ø], [ع], [œ], [a], [o], [o], [u] en fonction de leurs valeurs de F1, F2 et F3 de manière séparée. Notre objectif étant de vérifier dans quelle mesure les corrélats d'aperture, de position de la masse de la langue et des mouvements des lèvres étaient modifiés après les deux types d'entraînement. En outre, nous avons choisi d'évaluer les distances euclidiennes mesurées dans la charte de l'espace vocalique $\mathrm{F} 2 * \mathrm{~F} 3$ pour les voyelles $[\mathrm{u}] /[\mathrm{y}]$, $[\mathrm{e}] /[\varnothing]$ et $[\varepsilon] /[œ]$ afin de mesurer le degré de réalisation d'opposition entre ces voyelles [les voyelles antérieures labialisées sont notamment marquées (Wurzel 1998) et posent problème aux apprenants anglophones (Darcy et al. 2012)]. Les voyelles produites en contexte de disfluence, faux départs, hésitations ou mots inachevés ont été exclues de l'analyse. Le nombre final de segments analysés s'élevait donc à $2,1 \mathrm{k}$ voyelles.

\subsection{Extraction des valeurs formantiques}

Les valeurs spectrales des voyelles (F1-F2-F3) ont été extraites dans la zone médiane de chaque voyelle via un script Praat afin d'obtenir des valeurs formantiques plus ou moins stables. L'amplitude des pics a été détectée avec une bande inférieure à $5 \mathrm{kHz}$ pour les hommes et inférieure à $6 \mathrm{kHz}$ pour les femmes avec une fenêtre Gaussienne de $25 \mathrm{~ms}$. Dans le but d'exclure les valeurs formantiques aberrantes dues à la mauvaise détection de formants (voix craquée, assourdissements, globalisations etc.), nous avons employé un filtre selon Gendrot \& Decker (2007). Enfin, nous avons converti les valeurs formantiques $\mathrm{Hz}$ en Barks afin d'obtenir une normalisation par genre et par locuteur (Labov 2006)

\subsection{Distances euclidiennes}

A partir des valeurs formantiques obtenues en Barks dans la section précédente, nous avons calculé les distances euclidiennes sur le plan $\mathrm{F} 2 * \mathrm{~F} 3$ pour les paires de voyelles suivantes : $[\mathrm{u}] /[\mathrm{y}]$, $[\mathrm{e}] /[\varnothing]$ et $[\varepsilon] /[œ]$. Notre hypothèse était la suivante : une distance plus longue entre ces paires de voyelles sur le plan cartésien $\mathrm{F} 2 * \mathrm{~F} 3$ serait le résultat d'une tendance à catégoriser les voyelles en question, et, pour les paires $[\mathrm{e}] /[\varnothing]$ et $[\varepsilon] /[œ]$, traduirait plus particulièrement l'incidence des entraînements sur le trait de labialité. Autrement dit, si les participants bénéficient des avantages de l'une (ou des deux) méthodes, cela devrait avoir une incidence dans la position qu'occupent ces 
voyelles dans leur espace acoustique : plus elles sont éloignées dans la charte vocalique l'une par rapport à l'autre, plus cela marque une tendance à les opposer.

\section{Résultats}

Pour notre étude, nous avons modélisé les résultats obtenus moyennant des modèles mixtes avec le package lme 4 sous R. La puissance des variables prédictives (groupe, temps et voyelles) sur les variables dépendantes (F1, F2, F3, ainsi que les distances euclidiennes en question) a été estimée moyennant des tests du rapport de vraisemblance (likelihood ratio tests).

\subsection{Effets de la technique sur les valeurs formantiques}

Nous analysons ici les valeurs formantiques obtenues (F1, F2 et F3) avant et après (T1 et T2 respectivement) dans les deux groupes $(\mathrm{ART}=$ articulatoire vs $\mathrm{MVT}=$ verbo-tonale $)$. Les figures ci-dessous illustrent les espaces vocaliques sur le plan F1*F2 (Figure 1) et F2*F3 (Figure 2) selon les moyennes obtenues avant et après les traitements dans chacune des méthodes.
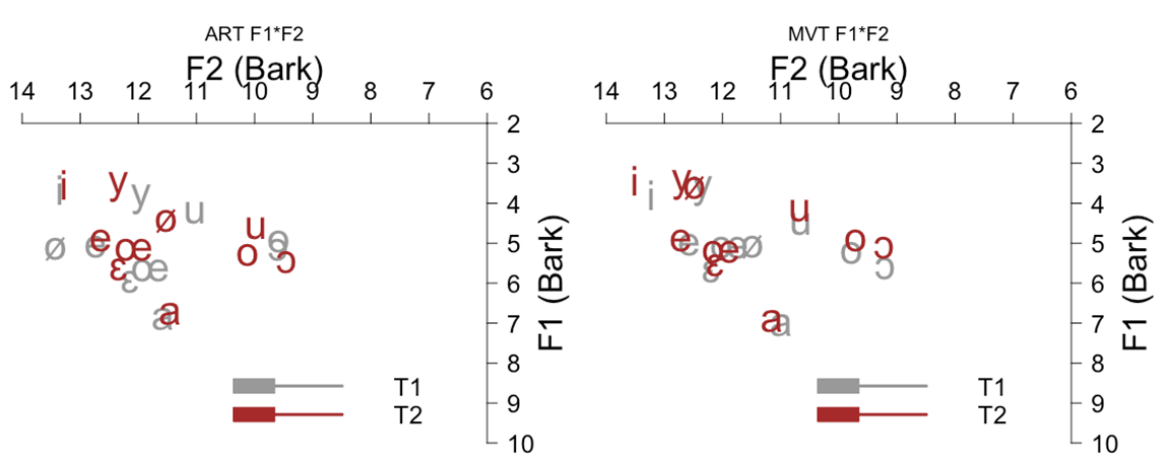

FIGURE 1 : Comparaison des triangles vocaliques sur le plan F1*F2 avant (T1) et après entraînement (T2) selon chacune des méthodes (Articulatoire vs Verbo Tonale).

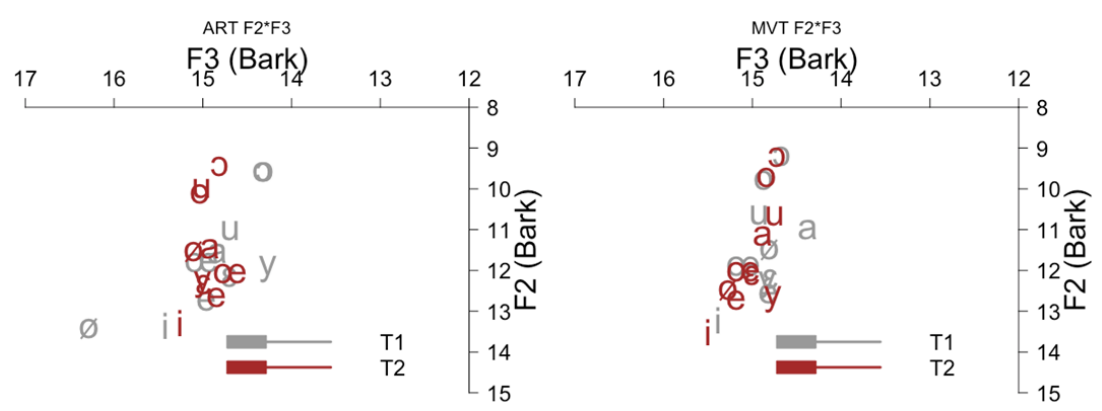

FIGURE 2 : Comparaison des triangles vocaliques sur le plan F2*F3 avant (T1) et après entraînement (T2) selon chacune des méthodes (Articulatoire vs Verbo Tonale).

Une inspection visuelle de ces espaces acoustiques ne nous permet pas de voir clairement les effets de chacune des méthodes, les voyelles se superposant entre elles dans la plupart des cas. Afin d'analyser l'impact des méthodes dans les valeurs formantiques des voyelles, nous avons construit plusieurs modèles (régressions linéaires à effets mixtes) avec «F1», «F2» et «F3» comme variables dépendantes, «Groupe » (ART vs MVT), «Temps» (T1 vs T2), «Voyelle» (le phonème en question) comme facteurs fixes (ainsi que leurs possibles interactions) et 
« Participants » comme variable aléatoire. Les résultats du modèle évaluant les effets du Groupe (T2 exclu) sur les trois variables dépendantes (F1, F2 et F3) n'ont pas atteint le seuil de significativité (toutes les valeurs de $p>0,05$ ), ce qui indique que les deux groupes étaient homogènes avant l'entrainement et donc comparables dans leur niveau de maîtrise phonique. En revanche, nous n'avons pas trouvé d'effet de Groupe, ni de Temps sur les valeurs formantiques de $\mathrm{F} 1$ et F2 (toutes les valeurs de $p>0,05$ ). Les résultats montrent que seules les valeurs de F3 sont affectées par le facteur Temps : elles sont plus élevées au T2 par rapport au T1 $(\beta=0.193$, e. t. $=$ $0,06, t=2,88, p<0,01)$, mais elles ne changent pas en fonction du Groupe $(p>0,05)$. En d'autres termes, les participants montrent une tendance à produire des valeurs de F3 plus élevées au T2 quelle que soit la technique employée. Tous ces résultants montrent qu'à l'exception de F3, les différences observées dans la figure ci-dessus sont certainement dues à la variabilité des participants, et qu'aucune des deux méthodes n'a d'incidence significative sur les valeurs formantiques de F1 et F2. Une analyse post-hoc par paires avec une correction de Tukey a montré que la voyelle [a] est en réalité la seule pour laquelle F3 est significativement différente entre T1 et T2 $(\beta=0,34$, é.-t. $=0,09, z=3,69, p<0,05)$. Une analyse individuelle par participant a montré une importante variabilité interlocuteur pour toutes les voyelles et a révélé que les valeurs de F3 peuvent être affectées différemment en fonction du temps. Ainsi, seulement 3 participants sur 8 ( 2 du groupe ART et $1 \mathrm{du}$ groupe MTV) produisent des valeurs de F3 plus basses au T2 pour la voyelle [œ] montrant ainsi que cette voyelle tend à être articulé avec un arrondissement des lèvres plus marqué après l'entrainement. Le reste des participants produisaient des valeurs de F3 plus hautes ou similaires. L'échantillon relativement petit de locuteurs analysés (4 par groupe) ne permet pas de tirer des conclusions définitives et plus de données seraient nécessaires pour une analyse statistique plus fiable.

\subsection{Effets de la technique sur les distances euclidiennes}

Notre deuxième objectif était de confirmer si les participants arrivaient à catégoriser les paires de phonèmes suivants $[\mathrm{u}] /[\mathrm{y}],[\mathrm{e}] /[\varnothing]$ et $[\varepsilon] /[\propto]$ en termes des distances euclidiennes dans l'espace F2*F3 (plus précisément, l'emploi de la dynamique des lèvres combinée à une articulation vocalique antérieure pour opposer ces phonèmes). Notre hypothèse était que, si des effets positifs de la méthode employée étaient observés, les participants devraient articuler ces segments avec une plus grande distance acoustique. La figure ci-dessous illustre les distances euclidiennes (en Barks) entre les phonèmes en question, avant et après entrainement, en fonction de la méthode. Cette figure illustre qu'il y a une certaine tendance à marquer des distances plus longues pour les phonèmes $[\mathrm{u}] /[\mathrm{y}]$, particulièrement avec la technique Articulatoire. En revanche, les résultats du modèle statistique montrent que ces différences ne sont pas significatives $(p>0,05)$. Cela suggère que ni le temps ni la méthode n'ont d'effet sur les distances euclidiennes entre ces paires de phonèmes produits par nos participants. Mais, encore une fois, l'échantillon limité de participants pourrait ne pas être en mesure de relever des différences. 


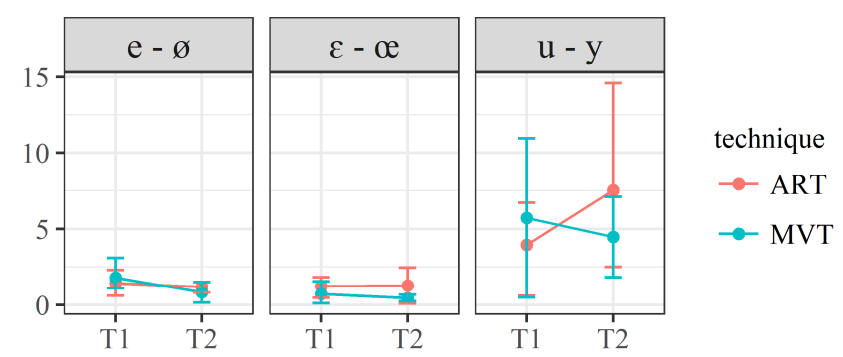

FIGURE 3 : Comparaison des triangles vocaliques sur le plan F2*F3 avant (T1) et après entraînement (T2) selon chacune des méthodes (Articulatoire vs Verbo Tonale).

\section{Discussion}

Dans cette étude, nous avons voulu comparer l'impact de deux méthodes de correction phonétique sur le développement du niveau segmental en L2. Nous pensions, comme pour la fluence dans une étude précédente, observer des différences entre les deux méthodes correctives choisies sur l'acquisition des voyelles. Nous avions formulé l'hypothèse selon laquelle la MVT permettrait une meilleure acquisition de F1 et F2. Pour tester cette hypothèse, nous avons analysé les valeurs F1, $\mathrm{F} 2$ et $\mathrm{F} 3$ de 2,1k segments vocaliques produits dans une tâche de lecture oralisée, avant et après entraînement à la correction phonétique, selon deux méthodes (Articulatoire vs. Verbo-Tonale). A partir des valeurs formantiques obtenues, nous avons également mesuré les distances euclidiennes sur le plan $\mathrm{F} 2 * \mathrm{~F} 3$ pour les paires de voyelles suivantes : $[\mathrm{u}] /[\mathrm{y}],[\mathrm{e}] /[\varnothing]$ et $[\varepsilon] /[œ]$, afin de déterminer si les apprenants avaient plus de facilités à catégoriser les voyelles données, avant et après entraînement, en fonction de la méthode.

Nos premiers résultats ne montrent pas d'incidence de l'apprentissage ni de la méthode sur les distances acoustiques ni sur les valeurs formantiques de F1 et F2. Les variations formantiques observées pourraient simplement être le résultat de la variabilité des participants. En revanche, nos résultats illustrent un effet du temps sur les valeurs formantiques de F3 pour la voyelle [a] chez tous les participants, après entrainement, quelle que soit la méthode utilisée. Les résultats issus des distances euclidiennes ne nous permettent cependant pas de dire si les participants arrivent à distinguer les paires de voyelles arrondies/non arrondies dans l'espace acoustique.

Une inspection individuelle par participant montre, par contre, que certains participants arrivent à baisser les valeurs formantiques de F3, indiquant ainsi que l'articulation de la voyelle [œ] est plus arrondie. Même si cela ne peut pas être généralisable, ces observations préliminaires nous amènent à penser que la correction phonétique des voyelles pourrait impliquer, que l'on en est conscience ou non, la prise en compte des mouvements des lèvres, faisant écho aux travaux sur le lien entre informations visuelles et information auditives en perception de la parole (voir par exemple : McGurk \& MacDonald 1976 ; Skipper et al, 2007 ; Yeung \& Werker, 2013). C'est d'autant plus intéressant que si la méthode articulatoire se focalise effectivement sur une description articulatoire des mouvements des différents articulateurs, la MVT utilise en revanche la gestualité accompagnatrice pour illustrer les mouvements articulatoires. D'après nos premiers résultats, il semblerait que la MVT ait des effets plus rapides dans l'amélioration de la composante prosodique que sur le plan segmental. En effet, en comparant les résultats positifs obtenus par Alazard (2013) sur la fluence avec les résultats reportés dans cette étude, il semblerait que l'acquisition des 
voyelles nécessite un entrainement plus long. Afin de confirmer ces premières tendances, nous envisageons, comme dans l'étude précédente de comparer les résultats acoustiques obtenus avec un test sur la base de jugements auditifs effectué auprès d'enseignants de FLE. Ces données préliminaires nous amènent également à réfléchir à la corporalité accompagnatrice non plus comme quelque chose de défini a priori en fonction de la méthode choisie mais comme quelque chose à adapter en fonction des phénomènes que l'on veut faire acquérir aux apprenants de L2. De nouvelles études sont donc nécessaires pour d'une part confirmer les tendances observées, en élargissant notamment l'analyse à l'acquisition des consonnes, et d'autre part, s'interroger sur l'impact de la gestualité (micro ou macro) sur l'acquisition de la phonétique en L2, en regardant aussi les effets obtenus avec d'autres méthodes de correction phonétique.

\section{$5 \quad$ Références}

AlazARD, C. (2013). Rôle de la prosodie dans la fluence en lecture oralisée chez des apprenants de Français Langue Etrangère. Thèse de doctorat, Université de Toulouse 2.

Bertrand, R., Blache, P., Espesser, R., Ferre, G., Meunier, C., Priego-Valverbe, B. \& RAUZY, S. (2008). Le CID-Corpus of Interactional Data-Annotation et exploitation multimodale de parole conversationnelle. Traitement automatique des langues, 49(3), 1-30.

BIGI, B. \& HIRST, D. (2012). Speech phonetization alignment and syllabification (SPPAS): a tool for the automatic analysis of speech prosody. Paper presented at the $6^{\text {th }}$ Speech Prosody Conference, Shanghai.

BILliERES, M. (2005). Les pratiques du verbo-tonal. Retour aux sources. In Berré, M.(Éd.), Linguistique de la parole et apprentissage des langues. Questions autour de la méthode verbotonale de P. Guberina. Mons: CIPA, 67-87.

BIRDSONG, D. (2003). Authenticité de prononciation en français L2 chez des apprenants tardifs anglophones : analyses segmentales et globales. Acquisition et interaction en langue étrangère 18, 17-36.

Boersma, P., \& WeENINK, D. (2005). Praat: doing phonetics by computer, http://www.praat.org

Bongaerts, T., Mennen, S., \& Slik, F.V.D. (2000). Authenticity of pronunciation in naturalistic second language acquisition: The case of very advanced late learners of Dutch as a second language. Studia linguistica 54, 298-308.

Darcy, I., Dekydtspotter, L., Sprouse, R. A., Glover, J., Kaden, C., McGuire, M., \& Scott, J. H. (2012). Direct mapping of acoustics to phonology: On the lexical encoding of front rounded vowels in L1 English-L2 French acquisition. Second Language Research, 28(1), 5-40.

GENDROT, C. \& ADDA-DECKER, M. (2007). Impact of duration and vowel inventory siez on formant values of oral vowels: an automated formant analysis from eight languages. Actes de 16th International Conference of Phonetic Sciences, 1417-1420.

LABOV, W. (2006). A sociolinguistic perspective on sociophonetic research. Journal of Phonetics, $34,500-515$. 
MCGuRK, H., \& MaCDonald, J. (1976). Hearing lips and seeing voices. Nature 264 (5588), 746748.

SkipPer, J. I., Van Wassenhove, V., Nusbaum, H. C., \& SMall, S. L. (2007). Hearing lips and seeing voices: how cortical areas supporting speech production mediate audiovisual speech perception. Cerebral cortex 17 (10), 2387-2399.

YEUNG, H. H., \& WERKER, J. F. (2013). Lip movements affect infant's audiovisual speech perception. Psychological Science 24 (5), 603-612.

WurZEL, W. U. (1998). On markedness. Theoretical Linguistics, 24 (1), 53-72. 